\title{
BMJ Open Healthy Lifestyle Program (HeLP) for low back pain: protocol for a randomised controlled trial
}

\author{
Emma K Robson, ${ }^{1,2,3}$ Steven J Kamper, ${ }^{3,4}$ Simon Davidson, ${ }^{1,2,3}$ \\ Priscilla Viana da Silva, ${ }^{1,2,3}$ Amanda Williams, ${ }^{1,2,3}$ Rebecca K Hodder, ${ }^{\oplus 1,2}$ \\ Hopin Lee, ${ }^{1,3,5}$ Alix Hall, ${ }^{1}$ Connor Gleadhill, ${ }^{1,2,3}$ Christopher M Williams ${ }^{\oplus, 2,3}$
}

To cite: Robson EK, Kamper SJ, Davidson S, et al. Healthy Lifestyle Program (HeLP) for low back pain: protocol for a randomised controlled trial. BMJ Open 2019;9:e029290. doi:10.1136/ bmjopen-2019-029290

- Prepublication history and additional material for this paper are available online. To view these files, please visit the journal online (http://dx.doi. org/10.1136bmjopen-2019029290).

Received 21 January 2019

Revised 30 June 2019

Accepted 04 July 2019

\section{Check for updates}

(c) Author(s) (or their employer(s)) 2019. Re-use permitted under CC BY-NC. No commercial re-use. See rights and permissions. Published by BMJ.

For numbered affiliations see end of article.

\section{Correspondence to} Dr Christopher M Williams; christopher.m.williams@ hnehealth.nsw.gov.au

\section{ABSTRACT}

Introduction Low back pain is one of the most common and burdensome chronic conditions worldwide. Lifestyle factors, such as excess weight, physical inactivity, poor diet and smoking, are linked to low back pain chronicity and disability. There are few high-quality randomised controlled trials that investigate the effects of targeting lifestyle risk factors in people with chronic low back pain. Methods and analysis The aim of this study is to determine the effectiveness of a Healthy Lifestyle Program (HeLP) for low back pain targeting weight, physical activity, diet and smoking to reduce disability in patients with chronic low back pain compared with usual care. This is a randomised controlled trial, with participants stratified by body mass index, allocated 1:1 to the HeLP intervention or usual physiotherapy care. HeLP involves three main components: (1) clinical consultations with a physiotherapist and dietitian; (2) educational resources; and (3) telephone-based health coaching support for lifestyle risk factors. The primary outcome is disability (Roland Morris Disability Questionnaire) at 26 weeks. Secondary outcomes include pain intensity, weight, quality of life and smoking status. Data will be collected at baseline, and at weeks 6, 12, 26 and 52. Patients with chronic low back pain who have at least one health risk factor (are overweight or obese, are smokers and have inadequate physical activity or fruit and vegetable consumption) will be recruited from primary or secondary care, or the community. Primary outcome data will be analysed by intention to treat using linear mixed-effects regression models. We will conduct three supplementary analyses: causal mediation analysis, complier average causal effects analysis and economic analysis.

Ethics and dissemination This study was approved by the Hunter New England Research Ethics Committee (Approval No 17/02/15/4.05), and the University of Newcastle Human Research Ethics Committee (Ref No $\mathrm{H}-2017-0222)$. Outcomes of this trial and supplementary analyses will be disseminated through publications in peer-reviewed journals and conference presentations. Trial registration number ACTRN12617001288314.

\section{INTRODUCTION}

Chronic low back pain is a worldwide public health problem with significant individual and economic burden. ${ }^{1-7}$ In 2016, low back

\section{Strengths and limitations of this study}

- The first randomised controlled trial investigating a comprehensive lifestyle intervention involving physiotherapy, dietetics and telephone health coaching for patients with chronic low back pain.

- The trial includes collection of a large range of variables to enable investigation of clinical effectiveness, cost-effectiveness and mechanisms of addressing lifestyle factors in patients with chronic low back pain to help guide healthcare policy decisions and clinical practice.

- Choice of primary and secondary outcomes is based on importance to patients with the condition.

pain was the leading cause of disability globally, accounting for over 57.6 million years lived with disability. ${ }^{1}$ Estimated total costs of low back pain are significant, with direct costs of care estimated at $£ 2.8$ billion in the $\mathrm{UK},{ }^{7}$ and $\$ 90$ billion in the USA. ${ }^{5}$ In Australia, annual costs exceed $\$ 4.8$ billion and low back pain reduces the country's gross domestic product by $\$ 2.9$ billion per year. ${ }^{3}$ Healthcare consumption, medication use, productivity losses and forced early retirement contribute to these costs. ${ }^{357}$

Lifestyle risk factors, including excess weight, physical inactivity, poor diet and smoking, contribute to the burden of low back pain. ${ }^{8-12}$ Meta-analysis of 33 studies found obesity was associated with increased prevalence of chronic low back pain (OR $1.4,95 \%$ CI 1.4 to 1.6 ) and increased care seeking for low back pain (OR 1.6, 95\% CI 1.5 to 1.7$).{ }^{8}$ Another meta-analysis of 27 studies demonstrated associations between smoking and chronic low back pain (OR 1.8, 95\% CI 1.3 to 2.5$).{ }^{11}$ Physical inactivity and poor diet contribute to obesity, and have an influence on chronic low back pain independently. ${ }^{13-18}$ Despite some inconsistency in the literature, evidence suggests low levels of physical 
activity are associated with chronic low back pain, ${ }^{13-15}$ as is diet-induced systematic inflammation. ${ }^{16} 17$

Preliminary clinical studies suggest addressing such lifestyle factors can improve low back pain outcomes including disability. ${ }^{12}{ }^{19-22}$ For example, a pre-post study of a weight loss programme targeting diet and physical activity showed significant improvement in pain-related disability in patients with low back pain. ${ }^{19}$ Increasing physical activity and providing exercise are widely accepted core treatment recommendations for chronic low back pain management ${ }^{2324}$ and reviews of the literature show exercise is effective in reducing disability (improvement of 2.5 points on 100-point scale, $95 \%$ CI 1.0 to 3.9 ) compared with controls. ${ }^{25}{ }^{26}$ In relation to smoking, one cohort study involving 5333 smokers with low back pain also showed clinically significant reductions in pain $(30 \%$ decrease) in those who quit smoking. ${ }^{20}$

Despite evidence suggesting the potential benefit of interventions targeting these lifestyle factors, ${ }^{21}{ }^{22}$ there is only one randomised controlled trial evaluating a lifestyle intervention for patients with chronic low back pain. ${ }^{27} 28$ This study found no effect, likely due to poor adherence to the treatment.

\section{Objective}

The primary aim of this trial is to determine the effectiveness of a multifocused Healthy Lifestyle Program (HeLP) for low back pain to reduce disability in patients with chronic low back pain, compared with usual physiotherapy care. Secondary objectives are to determine the effectiveness of the intervention on pain intensity, patient weight, quality of life and smoking status.

We will also conduct three supplementary analyses to assess: (1) treatment effect in those who complied with the intervention using complier average causal effects (CACE) analysis; (2) mediating effects of the intervention through pain, weight, smoking cessation, physical activity levels, diet, pain self-efficacy and psychological distress; (3) cost-effectiveness of the intervention from the health sector and societal perspectives.

\section{METHODS AND ANALYSIS \\ Study design}

The study will be a parallel-group pragmatic randomised controlled trial with two groups (figure 1). Participants will be stratified by body mass index (BMI) category and randomised 1:1 to either the HeLP intervention or usual physiotherapy care.

\section{Setting}

People with a main complaint of low back pain referred from primary care or secondary care at a major tertiary referral hospital in the Hunter New England Local Health District, New South Wales (NSW), or self-referred from community advertisements will be screened for eligibility.

\section{Eligibility}

Eligibility criteria are as follows:

- At least 18 years of age.

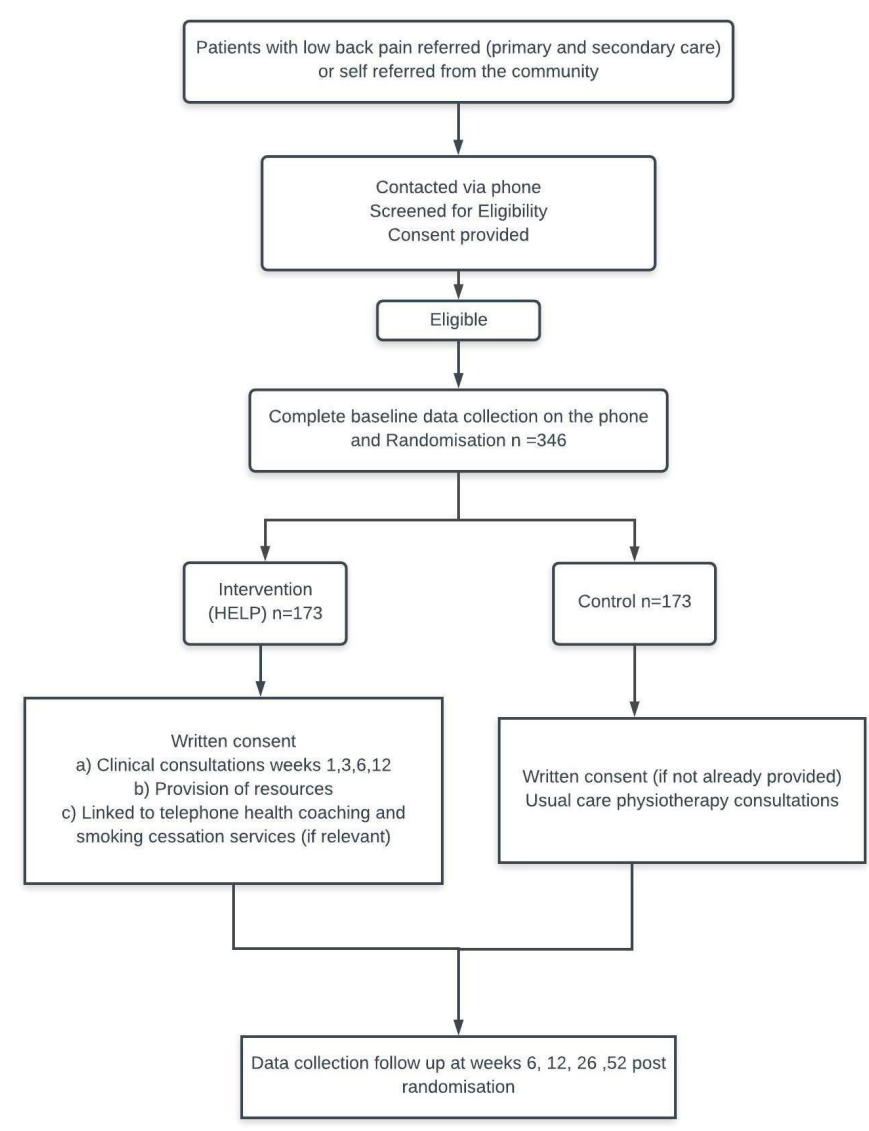

Figure 1 Planned flow of participants through the Healthy Lifestyle Program (HeLP) for low back pain trial.

- Chronic low back pain, defined as pain between the 12 th rib and buttock crease, with or without leg pain, of more than 3 months' duration. ${ }^{29}$

- Pain intensity rating over the last week of $\geq 3$ on an 11-point numerical rating scale where ' 0 ' represents no pain and ' 10 ' represents worst possible pain, ${ }^{30}$ or respond 'moderately', 'quite a bit' or 'extremely' when asked how much back pain interferes with completing normal daily activities of living (modified item of Short Form Health Survey-36 (SF-36)) ${ }^{30}$

- At least one health risk factor: overweight or obese $\left(\mathrm{BMI}>25 \mathrm{~kg} / \mathrm{m}^{2}\right){ }^{18}$ participate in less than $30 \mathrm{~min}$ of physical activity on 5 days of the week; ${ }^{31}$ current smoker; or eat less than two serves of fruits and five serves of vegetables per day, as a proxy of overall diet quality. $^{32}$

Exclusion criteria:

- Previous bariatric weight loss surgery.

- Currently undertaking weight loss or smoking cessation programme or care (eg, dietetics for weight loss, Jenny Craig, Lite n' Easy, Optifast, Weight Watchers, nicotine replacement therapy, Quitline).

- Back surgery in the previous 6 months or planned back surgery in the next 6 months.

- Known or suspected serious pathology causing back pain (ie, rheumatoid arthritis, cancer, fracture or infection). 
- Cannot actively engage in the intervention (unable to communicate, use a telephone or attend appointments, adapt meals or exercise).

- Comorbidity that does not allow safe completion of study procedures (eg, uncontrolled blood pressure or heart conditions, uncontrolled diabetes).

- Pregnant or planning pregnancy in the next 12 months.

\section{Details of the intervention and control}

Description of the trial design adheres to the Standard Protocol Items: Recommendations for Interventional Trials. ${ }^{33}$ The intervention and control conditions are described according to the Template for Intervention Description and Replication (online supplementary appendix 1$).{ }^{34}$

\section{Intervention (HeLP)}

Participants randomised to the intervention group will receive HeLP over a 6 -month period. The intervention draws on cognitive-behavioural therapy (CBT) and motivational interviewing (MI) to support pain management and facilitate positive behaviour change related to maintaining a healthy lifestyle (table 1 ).

HeLP includes:

1. Consultations with a physiotherapist and dietitian.

2. Provision of educational resources.

3. Telephone-based health coaching services for healthy lifestyle and smoking cessation (table 2).

\section{Consultations}

This component was codeveloped by a multidisciplinary team of investigators with expertise in physiotherapy, dietetics, psychology, nursing and health service management. Consultations aim to:

- Assess participants' back pain condition.

- Educate participants about back pain and lifestyle.

- Use behaviour change strategies to facilitate positive lifestyle changes (achieve a healthy body weight, increase physical activity, develop healthy eating habits, quit smoking).

- Initiate links with and engagement in supplementary support services.

- Reinforce positive behaviours and encourage self-regulation.

\section{Mode and timing of consultation delivery}

Participants will be offered to attend five clinical consultations of up to $60 \mathrm{~min}$ in duration; four with a registered physiotherapist, and one with a registered dietitian. Physiotherapy consultations will include an initial consultation and follow-up consultations at 3, 6 and 12 weeks after randomisation. The dietitian consultation will occur at week 3 , immediately after the physiotherapy consultation (table 2). Consultations will be conducted face to face; however, if patients are not able to attend appointments face to face they may be offered telehealth consultations (telephone or video conference) to deliver clinical content. The delivery method for all consultations will be recorded.

\section{Content (initial consultation)}

The initial consultation will involve three major components (table 1):

1. History and physical assessment. ${ }^{24}$

2. HeLP education (education about pain biology and links between pain and lifestyle).

3. Develop an individualised HeLP action plan to address lifestyle risks, incorporating behaviour change strategies and initiating a physical activity programme.

Physical assessment, education and advice are built on guideline recommendations and in line with expectations from physiotherapists of patients with back pain. ${ }^{23-25} 35-37$ Education and advice are designed to improve participants' understanding of the biopsychosocial nature of low back pain by presenting information on the nature of pain biology, ${ }^{38}$ address erroneous beliefs about back pain (eg, that low back pain is always associated with pathology) and outline links between low back pain and health behaviour risks (weight, smoking, and so on). Advice aims to encourage pain self-management, negotiate a physical activity plan and provide a platform for patients to engage with additional support services for healthy lifestyle.

Behaviour change strategies are based on principles of MI and CBT. ${ }^{37}{ }^{39-41}$ Evidence supports the use of CBT and MI in improving physical and behavioural outcomes in patients with back pain. ${ }^{37} 3942$ Behaviour change strategies include: assessing stage of change; goal setting with graded task assignment; and developing self-monitoring and regulation practices. Clinicians will work with patients to set tailored goals using graded task assignment, for example, agreeing on an initial goal of $10 \mathrm{~min}$ walking per day, to increase to $30 \mathrm{~min}$ per day by week 6. Clinicians will encourage self-monitoring and regulation through goal evaluation and use of patient resources to monitor pain, activity and eating patterns. Patient understanding of, and participation in, telephone health coaching services will also be encouraged.

\section{Content (follow-up consultations)}

Subsequent consultations (weeks 3, 6 and 12) will reinforce key messages, information and action plans provided in the initial consultation. Participants and clinicians will evaluate goals and progress, identify and address barriers to change and encourage self-monitoring (table 1).

The dietitian consultation will occur immediately after physiotherapy consult in week 3 . The consultation will focus on achieving or maintaining healthy eating behaviours to assist in weight management. Education and advice will be based on Australian healthy eating ${ }^{1832} 4344$ and obesity management guidelines. ${ }^{183243}$ Education will be tailored to participants' needs including information on recommended intake of the five key food groups, energy balance, portion sizes and general healthy lifestyle promotion. 
Table 1 Physiotherapy consultations

\begin{tabular}{|c|c|c|c|}
\hline Physiotherapy & Component & Content & Purpose \\
\hline \multirow[t]{3}{*}{ Week 1 (initial) } & Physical assessment & $\begin{array}{l}\text { Patient history and physical assessment } \\
\text { including assessing range of motion, evaluate } \\
\text { strength, flexibility, pain characteristics. } \\
\text { Collect anthropometric measurements (height, } \\
\text { weight). }\end{array}$ & $\begin{array}{l}\text { Develop rapport. } \\
\text { Assess and address patients' } \\
\text { expectations for physiotherapy } \\
\text { care. }\end{array}$ \\
\hline & Psychoeducation & $\begin{array}{l}\text { Introduce pain biology, concept of pain being } \\
\text { multifactorial and does not always equal } \\
\text { damage, as well as fluctuating nature of pain } \\
\text { conditions. } \\
\text { Acknowledgement that pain is real. } \\
\text { Discuss influence of lifestyle factors on back } \\
\text { pain and consequences of being overweight, } \\
\text { having a poor diet, inactivity, poor sleep and } \\
\text { smoking. } \\
\text { Introduce HeLP to support adoption of healthy } \\
\text { lifestyle behaviours. } \\
\text { Promote support services in the GHS and } \\
\text { Quitline. }\end{array}$ & $\begin{array}{l}\text { Correct erroneous pain beliefs, } \\
\text { increase knowledge, provide } \\
\text { rationale for need to change } \\
\text { health behaviour. }\end{array}$ \\
\hline & $\begin{array}{l}\text { Behaviour change } \\
\text { strategies }\end{array}$ & $\begin{array}{l}\text { Assess patients' stage of change and } \\
\text { motivations through questioning. } \\
\text { Acknowledge general barriers to lifestyle } \\
\text { change and programme adherence. } \\
\text { Establish and agree on commitment to } \\
\text { change. } \\
\text { Goal setting: establish patient management } \\
\text { and lifestyle goals. } \\
\text { Agree on graded exercises and physical } \\
\text { activity (eg, aim to start walking } 10 \text { min five } \\
\text { times a week). } \\
\text { Discuss strategies to facilitate self-monitoring } \\
\text { behaviours such as keeping activity, pain } \\
\text { and diet diaries and attending follow-up } \\
\text { appointments. }\end{array}$ & $\begin{array}{l}\text { Initiate process of behaviour } \\
\text { change, encourage safe } \\
\text { engagement in physical activity, } \\
\text { initiate engagement with } \\
\text { support services. }\end{array}$ \\
\hline \multirow[t]{2}{*}{ Weeks 3 and 6} & Psychoeducation & $\begin{array}{l}\text { Reinforce back pain education and lifestyle } \\
\text { messages in first consultation. }\end{array}$ & $\begin{array}{l}\text { Increase knowledge and } \\
\text { reinforce that pain also has } \\
\text { behavioural influences }\end{array}$ \\
\hline & $\begin{array}{l}\text { Behaviour change } \\
\text { strategies }\end{array}$ & $\begin{array}{l}\text { Evaluate goals and adapt with patient } \\
\text { discussion and assessment of diaries. } \\
\text { Problem solving: discuss patient barriers to } \\
\text { meeting goals and strategies to overcome. } \\
\text { Goal setting: adapt or progress graded } \\
\text { exercise and activity. } \\
\text { Discuss participation in the GHS and } \\
\text { Quitline services and encourage continued } \\
\text { participation (if appropriate). } \\
\text { Encourage continual encouragement of } \\
\text { self-monitoring. }\end{array}$ & $\begin{array}{l}\text { Reinforce positive behaviour, } \\
\text { support behaviour change and } \\
\text { self-monitoring behaviours. }\end{array}$ \\
\hline
\end{tabular}




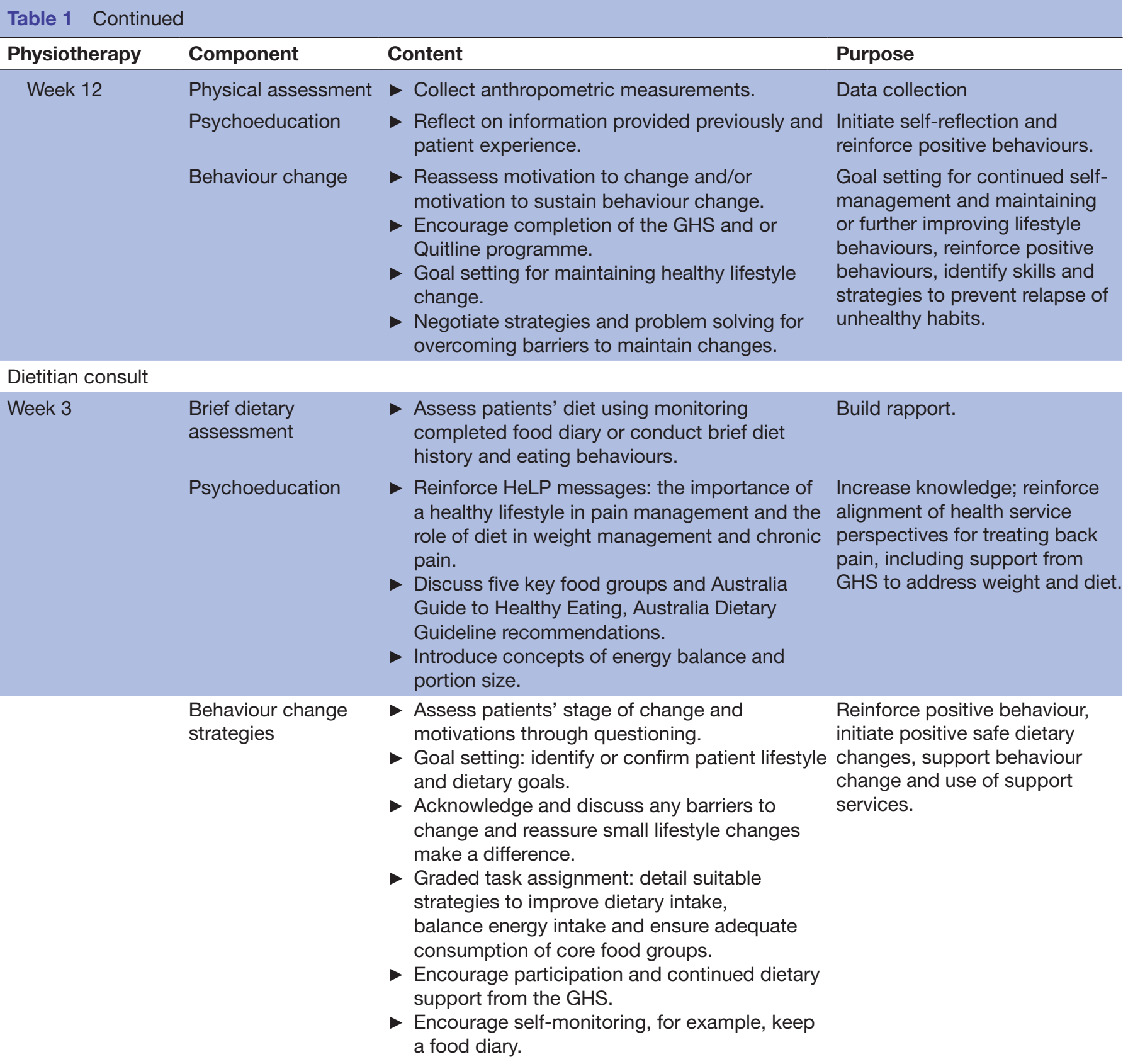

GHS, Get Healthy Service; HeLP, Healthy Lifestyle Program for Low Back Pain.

Clinicians will support and encourage patients to achieve their lifestyle goals and engage with telephone health coaching services. Goals and content of clinical care will be provided in the initial referrals to the telephone coaching services.

\section{Training for intervention delivery}

The intervention clinicians will attend multiple training sessions (including piloting the intervention) prior to study commencement and offered to attend a 2-day Health Behaviour Change course. ${ }^{45}$ Training involves background to the study, understanding intervention and appointment scheduling protocols for each consult, and behaviour change techniques. Clinicians will also learn how to use Research Electronic Data Capture (REDCap) ${ }^{46}$ an electronic data capture tool to record patient appointment attendance and delivery of intervention components. An intervention checklist will be provided to guide intervention delivery and optimise fidelity. Intervention clinicians will be provided implementation support via regular face-to-face and email contact with the research team for the duration of the study.

\section{Educational resources}

Patients will be provided with educational resources including an information booklet, access to a website, and a 5 min video ${ }^{47}$ reflecting education and key messages delivered in the consultations. The information booklet 


\begin{tabular}{|c|c|}
\hline Component & Week \\
\hline \multicolumn{2}{|l|}{ Intervention group } \\
\hline $\begin{array}{l}\text { Recruitment phone call: } \\
\text { eligibility screening, } \\
\text { baseline data collection and } \\
\text { randomisation }\end{array}$ & 0 \\
\hline Initial consultation & 1 \\
\hline $\begin{array}{l}\text { Referral to NSW GHS weight } \\
\text { management programme }\end{array}$ & $\begin{array}{l}\text { Following initial } \\
\text { consultation }\end{array}$ \\
\hline $\begin{array}{l}\text { NSW GHS weight management } \\
\text { programme* }^{*}\end{array}$ & $1-26$ \\
\hline Follow-up consultations & $3,6,12$ \\
\hline $\begin{array}{l}\text { Telephone interviews for } \\
\text { outcome assessments }\end{array}$ & $6,12,26,52$ \\
\hline $\begin{array}{l}\text { Referral to Quitline smoking } \\
\text { cessation programme }\end{array}$ & $\begin{array}{l}1-12 \text { based on patient } \\
\text { preference for timing }\end{array}$ \\
\hline $\begin{array}{l}\text { Quitline smoking cessation } \\
\text { programme }\end{array}$ & $\begin{array}{l}1-26 \text { (depending on } \\
\text { referral date) }\end{array}$ \\
\hline \multicolumn{2}{|l|}{ Control } \\
\hline $\begin{array}{l}\text { Recruitment phone call: } \\
\text { eligibility screening, } \\
\text { baseline data collection and } \\
\text { randomisation }\end{array}$ & 0 \\
\hline $\begin{array}{l}\text { Usual physiotherapy care } \\
\text { consultations }\end{array}$ & From week 1 \\
\hline $\begin{array}{l}\text { Telephone interviews for } \\
\text { outcome assessments }\end{array}$ & $6,12,26,52$ \\
\hline
\end{tabular}

*If patients miss calls, or put the programme temporarily on hold, the programme may run longer than 26 weeks. If participants achieve goals they may also graduate early after five calls. GHS, Get Healthy Service; NSW, New South Wales.

and website were codeveloped by back pain experts, intervention physiotherapists and dietitians. The booklet and website were piloted with consumers and assessed for cultural safety, and changes were made according to feedback. The information booklet and website detail pain education provided in the consults, healthy lifestyle information, information about support services and diaries to record goals and encourage self-monitoring. The $5 \mathrm{~min}$ video $^{47}$ will be sent to patients via short message service (SMS) or email, viewing will also be prompted at the week 3 appointment.

\section{Telephone health coaching}

\section{Healthy lifestyle coaching telephone service}

All patients in the intervention group will be referred to the telephone-based 'NSW Get Healthy Information and Coaching Service' immediately after their initial consultation (table 2).

The Get Healthy Service (GHS) is a telephone-based health coaching initiative provided by the NSW Government. The service aims to support people to modify unhealthy behaviours; increase physical activity levels; make healthy dietary changes; reduce alcohol consumption; and achieve and maintain a healthy body weight. ${ }^{48} 49$ There is evidence that the GHS is effective in reducing BMI, and waist circumference, and improves eating behaviours and physical activity in the general population. ${ }^{49} 50$

\section{Mode and timing of delivery}

The GHS involves up to 10 individually tailored phone calls over a 6-month period, delivered by qualified and specially trained health professionals. ${ }^{51}$ Calls are usually made bimonthly for the first 3 months $(n=6)$ to help facilitate behaviour change, with remaining calls tapering off for the following 3 months. Average call duration is 13 min. ${ }^{49}{ }^{50}$ Patients can graduate early from the GHS if they complete five coaching calls and meet their health goals.

\section{Content}

Content is based on the Australian Guide to Healthy Eating, and National Physical Activity Guidelines. ${ }^{31} 52$ Coaches provide education, advice and counselling to achieve or maintain a healthy weight through modifying energy intake, and use strategies to improve intake of the five key food groups and achieve moderate physical activity of $30 \mathrm{~min} 5$ days per week. The GHS aims to support, motivate and monitor the lifestyle goals patients established in their face-to-face consultations. Coaching uses MI techniques and self-regulation to support behaviour change. ${ }^{50}$

\section{Training for intervention delivery}

All GHS coaches are university-qualified health professionals (dietitians, exercise physiologists, health psychologists). All coaches involved in the trial will receive 3 hours of interactive face-to-face training, delivered by the principle investigators. Training is based on academic detailing, including educational outreach, technical assistance and provision of resources. Sessions include education about chronic pain and guideline-recommended care for patients with low back pain; current evidence for low back pain management and links to health behaviour risks and lifestyle; and advice from professional experiences treating patients, including common barriers to care and engagement in lifestyle management. Coaches are instructed how to make links between lifestyle behaviours, weight management and back pain care, and complete mock case studies to facilitate integration of the training into usual coaching practice. Coaches will be provided with resources and training material to guide learning, and for use with patients. Coaches will be provided with contact details (telephone and email) and encouraged to contact principle investigators for ad hoc support for guidance about challenges, cases or management questions.

\section{Smoking cessation programme}

Participants who identify as smokers at baseline will be referred to the NSW Quitline telephone smoking 
cessation programme. Referral to Quitline will occur within the first 12 weeks of the intervention (table 2), with the exact timing negotiated between the clinician and the patient, based on patient preference, stage of change and consent.

Quitline is a telephone counselling service aiming to encourage participants to set a quit date and facilitate successful quit attempts. An evaluation of the Australian Quitline services showed that $88 \%$ of callers sampled had made a quit attempt since their initial call, and of those who had made a quit attempt, $38 \%$ had ceased smoking at 6-month follow-up. Actual quit rates in those accessing Quitline after adjusting for non-responses were approximately $20 \% .^{53}$

\section{Mode and timing of delivery}

Those participating in Quitline receive an initial counselling call and are encouraged to set a quit date within the next month. Participants are offered a free counselling service of up to five calls from the day before the quit date to 30 days after the quit date. Call duration typically ranges from 10 to 20 min. $^{5455}$ 'Quit kits' containing information about quitting smoking are posted to participants upon enrolment in Quitline.

\section{Content}

Quitline counselling sessions include an assessment of smoking status, identification of triggers for smoking, coping strategies and information on effective quitting aids. Strategies to support quitting are tailored to individual preferences. 'Quit Kits' also include nicotine replacement therapies such as gum, lozenge and patches, guidance booklets and informational support.

\section{Training for intervention delivery}

All Quitline counsellors have a tertiary education qualification such as social work or psychology, and are trained in health education and counselling delivery using CBT and MI techniques.

\section{Control (usual care group)}

The usual care group will be referred for usual physiotherapy care in a public hospital outpatient physiotherapy clinic.

\section{Content}

The usual care appointments may include a physical assessment, and guideline-based advice and education for back pain. ${ }^{23} 24$

\section{Mode and timing of delivery}

Up to three face-to-face usual care appointments will be provided by a registered physiotherapist.

\section{Training for delivery}

Usual care physiotherapists will deliver their usual practices of care, however they will be asked not to provide specific support for lifestyle-related health behaviours. Physiotherapists will record components of usual care on a standardised form, entered directly into the REDCap database.

\section{Outcome assessment}

Patients will be asked to complete questionnaires at baseline (prior to randomisation), and at 6, 12, 26 and 52 weeks after randomisation (tables 2 and 3). Baseline data will be collected via telephone. At weeks 6, 12, 26 and 52 patients will have the option of providing data online (via an email or SMS link), on a paper-based form or transcribed by a trained telephone interviewer.

Objective height and weight will be collected at the initial consultation and weight will be measured again at 12 weeks (height measured at initial consultation only).

\section{Baseline demographic characteristics}

Baseline measures include: date of birth, gender, employment status, income, health insurance status, compensation status, previous episodes of back pain, duration of back pain and presence of coexisting medical conditions (eg, heart disease, stroke, diabetes, cancer, asthma, arthritis, depression).

\section{Outcome measures}

\section{Primary outcome}

The primary outcome is disability at 26 weeks measured via the Roland Morris Disability Questionnaire (RMDQ), a validated self-report 24-item low back pain-specific survey. ${ }^{56}$

\section{Key secondary outcomes}

Secondary outcomes include pain intensity, weight, measured according to International Society for the Advancement of Kinanthropometry protocols ${ }^{57}$ (week 1 at initial appointment and week 12 appointment) and via self-report (baseline, weeks 6, 12, 26 and 52); quality of life measured by the SF-12 version 2 and smoking status using NSW Population Health Survey questions; collected at all data collection time points. ${ }^{58}$

\section{Exploratory outcomes}

Exploratory outcomes, including potential mediators and cost outcomes, will be collected at all data collection time points (table 3).

\section{Process outcomes}

Process evaluation includes measurement of intervention fidelity via clinician completion of a checklist to record the delivery of intervention components in both groups, and by observation of a random $10 \%$ of intervention consultations. We will also measure: dose of intervention (number of consultations attended and number of support service calls received), supplementary interventions, programme completion and reasons for dropout.

\section{Recruitment procedures}

The recruitment process is detailed in the Consolidated Standards of Reporting Trials diagram (figure 1). Potentially eligible patients will be provided with a study 


\begin{tabular}{|c|c|c|}
\hline Domain & Measure & Time (weeks) \\
\hline \multicolumn{3}{|l|}{ Primary } \\
\hline $\begin{array}{l}\text { Disability } \\
\text { (endpoint } 26 \\
\text { weeks) }\end{array}$ & Roland Morris Disability Questionnaire (RMDQ) ${ }^{56}$ & $0,6,12,26,52$ \\
\hline \multicolumn{3}{|l|}{ Secondary } \\
\hline Pain intensity & $\begin{array}{l}11 \text {-point, } 0-10 \text { numerical rating scale as the average pain over the last week where } 0 \\
\text { indicates no pain and } 10 \text { indicates worst possible pain }{ }^{30}\end{array}$ & $0,6,12,26,52$ \\
\hline \multirow[t]{2}{*}{ Weight } & $\begin{array}{l}\text { Objective weight measured to the nearest } 0.1 \mathrm{~kg} \text { by a trained assessor using } \\
\text { International Society for the Advancement of Kinanthropometry (ISAK) procedures }{ }^{57}\end{array}$ & 1,12 \\
\hline & Self-reported weight $(\mathrm{kg})$ is also collected at all time points. & $0,6,12,26,52$ \\
\hline Quality of life & $\begin{array}{l}\text { 12-item Short Form Health Survey version } 2 \text { (0-100 scale; high score indicates greater } \\
\text { quality of life })^{58}\end{array}$ & $0,6,12,26,52$ \\
\hline Smoking status & $\begin{array}{l}2 \text { items from the NSW Health Survey (which describes your smoking status and how } \\
\text { many cigarettes smoked per day) }\end{array}$ & $0,6,12,26,52$ \\
\hline
\end{tabular}

\section{Exploratory outcomes}

Physical activity International Physical Activity Questionnaire (IPAQ) reported as average hours and minutes spent participating in moderate to vigorous activity ${ }^{63}$

$0,6,12,26,52$

\begin{tabular}{|c|c|c|}
\hline Nutrition & $\begin{array}{l}\text { 21-item Food Frequency Questionnaire of intake over the past month (response options } \\
\text { for fruits, vegetables, discretionary choices, wholegrains and dairy categories: rarely or } \\
\text { never, less than once a week, once a week, } 2-3 \text { times a week, 4-6 times a week, 1-2 } \\
\text { times a day, 3-4 times a day, 5+ a day, and response options for meat categories: rarely } \\
\text { or never, less than once a week, once a week, 2-3 times a week, 4-6 times a week, } 7+ \\
\text { times a week) }\end{array}$ & $0,6,12,26,52$ \\
\hline Sleep quality & $\begin{array}{l}\text { Item } 6 \text { from the Pittsburgh Sleep Quality Index (response options: very bad, fairly bad, } \\
\text { fairly good, very good) }{ }^{65}\end{array}$ & $0,6,12,26,52$ \\
\hline Pain self- efficacy & $\begin{array}{l}\text { 2-item validated Pain Self-Efficacy Questionnaire (PSEQ-2) on a scale of 0-6 with } 0 \\
\text { indicating not at all confident and } 6 \text { completely confident }{ }^{66}\end{array}$ & $0,6,12,26,52$ \\
\hline $\begin{array}{l}\text { Psychological } \\
\text { distress }\end{array}$ & $\begin{array}{l}\text { Kessler } 6 \text { Questionnaire as how often a feeling was experienced over the past } 30 \text { days } \\
\text { (response options: all of the time, most of the time, some of the time, a little of the time, } \\
\text { none of the time) }{ }^{67}\end{array}$ & $0,6,12,26,52$ \\
\hline $\begin{array}{l}\text { Alcohol } \\
\text { consumption }\end{array}$ & $\begin{array}{l}\text { Alcohol Use Disorders Identification Test (AUDIT-C) (0-12 scale) high score greater risk } \\
\text { of alcohol-related harm } 68\end{array}$ & $0,6,12,26,52$ \\
\hline
\end{tabular}

Process and economic measures

\begin{tabular}{|c|c|c|}
\hline Adverse events & $\begin{array}{l}\text { Open-text question: 'Have you developed any new medical conditions or an } \\
\text { exacerbation of an existing condition?' }\end{array}$ & 6,52 \\
\hline $\begin{array}{l}\text { Health } \\
\text { economics }\end{array}$ & $\begin{array}{l}\text { Self-reported health and home care utilisation and medication use. Intervention costs: } \\
\text { staff time, phone calls, referral and written materials. GHS and Quitline costs: number } \\
\text { and call duration. Self-reported work absenteeism, presenteeism. }\end{array}$ & $0,6,12,26,52$ \\
\hline
\end{tabular}

GHS, Get Healthy Service; NSW, New South Wales.

information letter. The letter details the study procedures and informs patients they will be contacted by the research team to discuss participation. When patients are called, research personnel will screen for eligibility.

Eligible patients will verbally consent to participation and have baseline data collected over the phone. Written consent will be obtained at the initial consultation.

\section{Randomisation procedures}

Eligible consenting patients will complete baseline data collection over the phone with a trained interviewer, and be randomised via a concealed central randomisation service. Patients will be randomly allocated to the intervention or control group (1:1 ratio). A permuted 6:4 block randomisation approach will be used so that the distribution of healthy weight, overweight and obese participants is equal across treatment conditions (intervention or control). Interviewers will be prompted by the system to input BMI category (healthy weight $\leq 24.9 \mathrm{~kg} / \mathrm{m}^{2}$, overweight $\geq 25$ to $<30 \mathrm{~kg} / \mathrm{m}^{2}$, obese $\geq 30 \mathrm{~kg} / \mathrm{m}^{2}$ ) within the REDCap database which allocates participants to an experimental group based on a prespecified randomisation schedule generated by an independent statistician. 


\section{Blinding}

On study entry participants will be told that they will receive one of two treatment programmes delivered by a physiotherapist. To reduce ascertainment bias, participants are not informed of the specific treatment details before allocation, nor provided details about the treatment to which they are not assigned. To reduce performance bias, the clinicians involved in the study deliver only the intervention, or the control. It is not possible for objective anthropometry outcomes to be blinded, as intervention and control physiotherapists will perform the measurements at the consults. Statisticians will conduct analyses blinded to treatment allocation using dummy coding for treatment group. Analyses will be conducted according to a prespecified, published analysis plan. Treatment group will be unblinded at the completion of analysis.

\section{Data analysis}

Data will be analysed using the intention-to-treat principle with the number of analyses restricted and specified a priori. Data integrity will be monitored by regularly scrutinising data files for omissions and errors. All manually entered data will be double-checked.

\section{Sample size}

Sample size was calculated using the method of Twisk for mixed models, with four repeated observations, an estimated intracluster correlation (correlation between the observations) of 0.5 , alpha of $5 \%$ and allowing for up to $18 \%$ loss to follow-up. A total of 346 patients (173 per group) provide over $80 \%$ power to detect between-group differences of 3 points (SD 5) on $\mathrm{RMDQ}^{59}$ at 26 weeks. This is the smallest worthwhile effect that would justify the implementation of the intervention. We ignored the increase in statistical power due to stratification and including baseline covariates in the analysis.

\section{Effect estimates}

A linear mixed-effects regression model will be used to estimate the effectiveness of the intervention in reducing self-reported disability compared with control using all data points over 26 weeks of follow-up. A time by treatment group interaction term will be included as a fixed effect to assess the between-group differences in adjusted mean disability scores at each time point. Baseline disability scores will be included as a fixed effect in the model. Treatment group, time, BMI category and potential confounders (participant characteristics) will also be included as fixed effects. A random subject-level intercept will be used to account for the repeated measures of the data. If more than $10 \%$ of the data are missing the pattern of missing data will be examined and an appropriate method of multiple imputation (depending on the data) will be used. Sensitivity analysis will involve comparing complete case to imputed data analyses. A secondary analysis will explore the differential effects of the intervention on primary outcome for normal weight versus overweight/obese. The sample size provides sufficient power to detect a differential effect of 2 points on the RMDQ between normal weight and overweight/obese patients. This question will be evaluated using a linear mixed-effects model including a three-way fixed effect interaction term: treatment group, time and BMI category.

We prespecify four key secondary outcomes for interpretation to reduce the possibility of type I error. Treatment effects will be estimated using linear mixed-effects and logistic mixed-effects regression models. Adjusted mean differences (continuous variables) or differences in proportions (dichotomous variables) will be assessed for each outcome at each follow-up point, with 26 weeks after randomisation being considered the primary endpoint of the study. Exploratory analyses will be conducted on outcomes of health behaviours, self-efficacy and psychological distress. The same fixed and random effects prespecified for the main outcome model will be used in all secondary and exploratory analyses.

\section{Supplementary analyses}

Three preplanned supplementary analyses will be undertaken. We will publish detailed protocols on open science repositories prior to unblinding of data. Analyses will include:

- CACE analyses will be conducted for the primary and key secondary outcomes. ${ }^{60}$ The threshold for compliance will be attendance at least two intervention consultations and five or more completed GHS telephone calls (unless earlier graduation from the GHS programme).

- Causal mediation analysis will be used to investigate treatment mechanisms using data collected at baseline, and 12 and 26 weeks. ${ }^{61}$ We will assess the mediating effects of physical activity, diet, pain, self-efficacy, weight and symptoms of psychological distress on low back pain disability.

Three economic analyses will be conducted. First, a cost model comparing the costs associated with the control and intervention pathways. Second, a cost-utility analysis from the perspective of the health sector including individual healthcare utilisation costs (healthcare service and medication use). Third, a cost-utility analysis from a societal perspective including the additional non-healthcare costs (eg, carer or community service costs) and costs associated with work absenteeism and presenteeism. Costs will be based on standard published rates and self-reported (out-of-pocket) costs. Health state utilities (to estimate quality-adjusted life years) will be obtained from SF-12 score and transformed into health state utilities via the SF-6D algorithm.

\section{Patient and public involvement}

Development of the research question and the intervention design was based on previously conducted randomised controlled trials and data from patients on the orthopaedic surgical waitlist at the John Hunter Hospital, Newcastle, Australia, in 2014. ${ }^{2862}$ Patients were involved in pilot stages of the study to provide feedback 
on physiotherapy care, health coaching and resources. Patients will not be involved in recruitment of participants or conduct of the study. Results of this study will be available to the public and patients and published in open access peer-reviewed journals.

\section{Ethics and dissemination}

Outcomes of this trial and supplementary analyses will be disseminated through publications in peer-reviewed journals and conference presentations.

\section{Author affiliations}

${ }^{1}$ School of Medicine and Public Health, Hunter Medical Research Institute, The University of Newcastle, Callaghan, Newcastle, Australia

${ }^{2}$ Hunter New England Population Health, Wallsend, New South Wales, Australia ${ }^{3}$ Centre for Pain, Health and Lifestyle, Sydney, New South Wales, Australia ${ }^{4}$ School of Public Health, University of Sydney, Sydney, New South Wales, Australia ${ }^{5}$ Department of Orthopaedics, Rheumatology and Musculoskeletal Sciences, University of Oxford Nuffield, Oxford, UK

Acknowledgements The authors thank the Physiotherapy Department at the Royal Newcastle Centre, John Hunter Hospital, for assisting in the intervention delivery. The authors also thank the Hunter New England Population Health Computer Assisted Telephone Interviewing (CATI) team for undertaking recruitment and data collection and the wider Hunter Population Health facility for provision of necessary resources.

Contributors CMW and SJK designed the project and secured funding, contributed to ethical approval, design of consultation content, trained relevant research staff, development of patient resources, data collection tools, procedures and databases; contributed to statistical analysis protocol, contributed to drafting the manuscript, critical revision of intellectual content and approved the final version. EKR, SD, PVS $\mathrm{CG}$ and $\mathrm{HL}$ contributed to ethical approval, design of consultation content, trained relevant research staff, development of patient resources, data collection tools, procedures and databases; contributed to statistical analysis protocol; delivery of the intervention; and contributed to drafting the manuscript, critical revision of intellectual content and approved the final version. AW and RKH contributed to ethical approval, design of consultation content, trained relevant research staff, development of patient resources, data collection tools, procedures and databases; and critical revision of intellectual content and approved the final version of the protocol. AH contributed to development of data collection tools, procedures and databases; developed the randomisation schedule and statistical analysis protocol; and contributed to drafting the manuscript, critical revision of intellectual content and approved the final version.

Funding This work was supported by a National Health and Medical Research Council (NHMRC) project grant (Grant No APP1100992).

Competing interests None declared.

Patient consent for publication Not required.

Ethics approval This study was approved by the Hunter New England Research Ethics Committee (Approval No 17/02/15/4.05), and the University of Newcastle Human Research Ethics Committee (Ref No H-2017-0222).

Provenance and peer review Not commissioned; externally peer reviewed.

Open access This is an open access article distributed in accordance with the Creative Commons Attribution Non Commercial (CC BY-NC 4.0) license, which permits others to distribute, remix, adapt, build upon this work non-commercially, and license their derivative works on different terms, provided the original work is properly cited, appropriate credit is given, any changes made indicated, and the use is non-commercial. See: http://creativecommons.org/licenses/by-nc/4.0/.

\section{REFERENCES}

1. Vos T, Abajobir AA, Abate $\mathrm{KH}$, et al. Global, regional, and national incidence, prevalence, and years lived with disability for 328 diseases and injuries for 195 countries, 1990-2016: a systematic analysis for the global burden of disease study 2016. The Lancet 2017;390:1211-59.
2. Hoy D, March L, Brooks P, et al. The global burden of low back pain: estimates from the global burden of disease 2010 study. Ann Rheum Dis 2014;73:968-74.

3. Schofield DJ, Shrestha RN, Percival R, et al. The personal and national costs of early retirement because of spinal disorders: impacts on income, taxes, and government support payments. The Spine Journal 2012;12:1111-8.

4. Henschke N, Kamper SJ, Maher CG. The epidemiology and economic consequences of pain. Mayo Clin Proc 2015;90:139-47.

5. Dagenais S, Caro J, Haldeman S. A systematic review of low back pain cost of illness studies in the United States and internationally. The Spine Journal 2007:8-20.

6. Arthritis and osteoporosis Victoria. A problem worth solving: the rising cost of musculoskeletal condtions in Australia; 2013.

7. Hong J, Reed C, Novick D, et al. Costs associated with treatment of chronic low back pain: an analysis of the UK general practice research database. Spine 2013;38:75-82.

8. Shiri R, Karppinen J, Leino-Arjas P, et al. The association between obesity and low back pain: a meta-analysis. Am J Epidemiol 2010;171:135-54.

9. Heuch I, Heuch I, Hagen K, et al. Body mass index as a risk factor for developing chronic low back pain: a follow-up in the Nord-Trondelag health study. Spine 2013;38:133-9.

10. Suri P, Boyko EJ, Smith NL, et al. Modifiable risk factors for chronic back pain: insights using the co-twin control design. Spine $J$ 2017;17:4-14.

11. Shiri R, Karppinen J, Leino-Arjas P, et al. The association between smoking and low back pain: a meta-analysis. Am J Med 2010;123:87.e7-87.e35.

12. Bohman T, Alfredsson L, Jensen I, et al. Does a healthy lifestyle behaviour influence the prognosis of low back pain among men and women in a general population? a population-based cohort study. BMJ Open 2014;4:e005713.

13. Lin C-WC, McAuley JH, Macedo L, et al. Relationship between physical activity and disability in low back pain: a systematic review and meta-analysis. Pain 2011;152:607-13.

14. Heneweer H, Vanhees L, Picavet SJH. Physical activity and low back pain: a U-shaped relation? Pain 2009;143:21-5.

15. Shiri R, Falah-Hassani K. Does leisure time physical activity protect against low back pain? systematic review and meta-analysis of 36 prospective cohort studies. Br J Sports Med 2017;51:1410-8.

16. Seaman DR. The diet-induced proinflammatory state. J Manipulative Physiol Ther 2002;25:168-79.

17. Macphail K. C-Reactive protein, chronic low back pain and, diet and lifestyle. Int Musculoskelet Med 2015;37:29-32.

18. National Health and Medical Research Council. Clinical practice guidelines for the management of overweight and obesity in adults, adolescents and children in Australia. Melbourne: National Health and Medical Research Council, 2013.

19. Roffey DM, Ashdown LC, Dornan HD, et al. Pilot evaluation of a multidisciplinary, medically supervised, nonsurgical weight loss program on the severity of low back pain in obese adults. Spine $J$ 2011;11:197-204.

20. Behrend C, Prasarn M, Coyne E, et al. Smoking cessation related to improved patient-reported pain scores following spinal care. J Bone Joint Surg Am 2012;94:2161-6.

21. Dean $E$, Söderlund $A$. What is the role of lifestyle behaviour change associated with non-communicable disease risk in managing musculoskeletal health conditions with special reference to chronic pain? BMC Musculoskelet Disord 2015;16:87.

22. Wai EK, Rodriguez S, Dagenais S, et al. Evidence-Informed management of chronic low back pain with physical activity, smoking cessation, and weight loss. The Spine Journal 2008;8:195-202.

23. Wong JJ, Côté P, Sutton DA, et al. Clinical practice guidelines for the noninvasive management of low back pain: a systematic review by the Ontario protocol for traffic injury management (optima) collaboration. Eur J Pain 2017;21:201-16.

24. National Institute for Health and Care Excellence (NICE). Low back pain and sciatica in over 16S: assessment and management; 2016.

25. Gordon R, Bloxham S. A systematic review of the effects of exercise and physical activity on non-specific chronic low back pain. Healthcare 2016;4:E22.

26. Hayden JA, van Tulder MW, Malmivaara A, et al. Exercise therapy for treatment of non-specific low back pain. Cochrane Database Syst Rev 2005;(3):CD000335.

27. Williams A, Wiggers J, O'Brien KM, et al. A randomised controlled trial of a lifestyle behavioural intervention for patients with low back pain, who are overweight or obese: study protocol. BMC Musculoskelet Disord 2016;17. 
28. Williams A, van Dongen JM, Kamper SJ, et al. Economic evaluation of a healthy lifestyle intervention for chronic low back pain: a randomized controlled trial. Eur J Pain 2019;23:621-34.

29. Krismer M, van Tulder M, Low Back Pain Group of the Bone and Joint Health Strategies for Europe Project, et al. Strategies for prevention and management of musculoskeletal conditions. low back pain (non-specific). Best Pract Res Clin Rheumatol 2007;21:77-91.

30. Hawker GA, Mian S, Kendzerska T, et al. Measures of adult pain: visual analog scale for pain (vas pain), numeric rating scale for pain (NRS pain), McGill pain questionnaire (MPQ), short-form McGill pain questionnaire (SF-MPQ), chronic pain grade scale (CpGs), short Form-36 bodily pain scale (SF). Arthritis Care Res 2011;63:S240-S252.

31. Australian Government Department of Health. Australia's physical activity and sedentary behaviour guidelines for adults; 2017.

32. National Health and Medical Research Council. Australian dietary guidelines. Canberra: National Health and Medical Research Council, 2013.

33. Chan A-W, Tetzlaff JM, Gøtzsche PC, et al. Spirit 2013 explanation and elaboration: guidance for protocols of clinical trials. BMJ 2013;346:e7586.

34. Hoffmann TC, Glasziou PP, Boutron I, et al. Better reporting of interventions: template for intervention description and replication (TIDieR) checklist and guide. BMJ 2014;348.

35. Verbeek J, Sengers M-J, Riemens L, et al. Patient expectations of treatment for back pain: a systematic review of qualitative and quantitative studies. Spine 2004;29:2309-18.

36. Kamper SJ, Haanstra TM, Simmons K, et al. What do patients with chronic spinal pain expect from their physiotherapist? Physiother Can 2018;70:36-41.

37. Sveinsdottir V, Eriksen HR, Reme SE. Assessing the role of cognitive behavioral therapy in the management of chronic nonspecific back pain. J Pain Res 2012;5:371-80.

38. Moseley GL, Butler DS. Fifteen years of explaining pain: the past, present, and future. J Pain 2015;16:807-13.

39. Vong SK, Cheing GL, Chan F, et al. Motivational enhancement therapy in addition to physical therapy improves motivational factors and treatment outcomes in people with low back pain: a randomized controlled trial. Arch Phys Med Rehabil 2011;92:176-83.

40. Hall K, Gibbie T, Lubman DI. Motivational interviewing techniques - facilitating behaviour change in the general practice setting. Aust Fam Physician 2012;41:660-7.

41. British Association for Behvioural and Cognitive Psychotherapies. What is CBT? 2012.

42. Henschke N, Ostelo RWJG, van Tulder MW, et al. Behavioural treatment for chronic low-back pain. Cochrane Database Syst Rev 2010;49.

43. Australian Government national Health and Medical Research Council Department of Health and Ageing. Australian guide to healthy eating.

44. National Health and Medical Research Council. Summary guide for the management of overweight and obesity in primary care. Melbourne: National Health and Medical Research Council, 2013.

45. Health Change Australia. HealthChange $\AA$ methodology and training options; 2018.

46. Harris PA, Taylor R, Thielke R, et al. Research electronic data capture (REDCap)--a metadata-driven methodology and workflow process for providing translational research informatics support. $J$ Biomed Inform 2009;42:377-81.

47. Mosely L, Moen D. Tame the beast: its time to rethink persistant pain.

48. O'Hara BJ, Phongsavan P, Eakin EG, et al. Effectiveness of Australia's get healthy information and coaching service: maintenance of self-reported anthropometric and behavioural changes after program completion. BMC Public Health 2013;13:175.

49. O'Hara BJ, Phongsavan P, Venugopal K, et al. Effectiveness

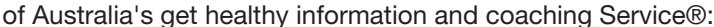
translational research with population wide impact. Prev Med 2012;55:292-8.

50. O'Hara BJ, Gale J, McGill B, et al. Weight-Related goal setting in a Telephone-Based preventive Health-Coaching program: demonstration of effectiveness. Am J Health Promot 2017;31:491-501.

51. O'Hara BJ, Phongsavan P, Rissel C, et al. Role of general practice in the utilisation of the NSW get healthy information and coaching service. Aust J Prim Health 2015;21:182-8.

52. Brown W, Bauman A, Bull F. Development of Evidence - based Physical Activity Recommendations for Adults (18-64 years) Report prepared for the Australian Government Department of Health; 2012. Content/health-pubhlth-strateg-phys-act-guidelies/\$File/DEB-PARAdults-18-64years.pdf

53. Greenhalgh E, Stillman S, Ford C. Cessation assistance: Telephoneand Internet-based interventions Tobacco in Australia: Facts and issues Melbourne: Cancer Council Victoria; 2016.

54. Tzelepis F, Paul CL, Wiggers J, et al. A randomised controlled trial of proactive telephone counselling on cold-called smokers' cessation rates. Tob Control 2011;20:40-6.

55. NSW Government Cancer Insititute NSW. Nsw Quitline; 2017.

56. Roland M, Morris R. A study of the natural history of back pain. Part I: development of a reliable and sensitive measure of disability in lowback pain. Spine 1983;8:141-4.

57. International Society for the advancement of Kinanthropometry. International standards for Antrhopometric assessment. Underdale ISAK; 2001

58. Ware J, Kosinski M, Turner-bowker D, et al. User's Manual for the SF$12 v 2$ Health Survey (With a Supplement Documenting SF-12 Health Survey. QualityMetric Incorporated: Lincoln, 2002.

59. Maughan EF, Lewis JS. Outcome measures in chronic low back pain Eur Spine J 2010;19:1484-94.

60. Dunn G, Maracy M, Tomenson B. Estimating treatment effects from randomized clinical trials with noncompliance and loss to follow-up: the role of instrumental variable methods. Stat Methods Med Res 2005;14:369-95.

61. Lee GM H, McAuley JH, Kamper SJ, et al. Causal mechanisms in the clinical course and treatment of back pain. Best Practice \& Research Clinical Rheumatology 2017.

62. O'Brien KM, Wiggers J, Williams A, et al. Telephone-based weight loss support for patients with knee osteoarthritis: a pragmatic randomised controlled trial. Osteoarthritis Cartilage 2018;26:485-94.

63. Craig CL, Marshall AL, Sjöström M, et al. International physical activity questionnaire: 12 -country reliability and validity. Med Sci Sports Exerc 2003;35:1381-95.

64. Cleghorn CL, Harrison RA, Ransley JK, et al. Can a dietary quality score derived from a short-form FFQ assess dietary quality in UK adult population surveys? Public Health Nutr 2016;19:2915-23.

65. Buysse DJ, Reynolds CF, Monk TH, et al. The Pittsburgh sleep quality index: a new instrument for psychiatric practice and research. Psychiatry Res 1989;28:193-213.

66. Nicholas MK, McGuire BE, Asghari A. A 2-item short form of the pain self-efficacy questionnaire: development and psychometric evaluation of PSEQ-2. J Pain 2015;16:153-63.

67. Kessler RC, Barker PR, Colpe LJ, et al. Screening for serious mental illness in the general population. Arch Gen Psychiatry 2003;60:184-9.

68. Bush K, Kivlahan D, McDonell M, et al. The audit Acohol consumption questions (AUDIT-C): a brief screening test for problem drinking. Arch Internal Med 1998;3:1789-95. 